\title{
Medium and long-term prognosis of transcatheter aortic valve implantation from the perspective of left ventricular diastolic function
}

\author{
Satoru Kayama ${ }^{1}$, Shungo Aratake ${ }^{1}$, Shigehito Sawamura ${ }^{1}$, \\ Yusuke Watanabe ${ }^{2}$, Ken Kozuma ${ }^{2}$ \\ ${ }^{1}$ Department of Anesthesia, Teikyo University School of Medicine, Japan \\ ${ }^{2}$ Department of Cardiology, Teikyo University School of Medicine, Japan
}

\begin{abstract}
Background: The effects of left ventricular (LV) diastolic function are well known in cardiac surgery, but unclear in transcatheter aortic valve implantation (TAVI). The objective of this study was to examine the association of preoperative LV diastolic function with medium to long-term outcomes of TAVI. Methods: Eighty patients who underwent TAVI were classified into grades I, II and III based on preoperative LV diastolic function. Findings related to cardiovascular outcomes after TAVI were extracted retrospectively from clinical and echocardiographic data and relationships with diastolic function were examined.

Results: The average follow-up was 529 days (interquartile range $\{I Q R] 358-741$ days). Cardiovascular events occurred in 17 cases, including 6 deaths, and were significantly associated with Euro II score $(p=0.043)$, albumin level $(p=0.026)$, coronary artery disease $(C A D)(p=0.017)$, and diastolic function $(p<0.001)$. The 360 -day event-free rates were $89.5 \%$, $89.5 \%$ and $37.5 \%$ for grades I, II and III ( $p=0.00013)$. Median event-free survival (EFS) in grade III cases was 180 days. In a Cox proportional hazard model, LV diastolic dysfunction (hazard ratio [HR] 3.99, 95\% confidence interval [CI] $1.35-11.80, p=0.012)$ and low albumin (HR 4.73, 95\% CI 1.42-15.80, $p=0.012)$ were significant independent predictors of reduced EFS.

Conclusions: Medium to long-term outcomes of TAVI were poorer in patients with deteriorated LV diastolic function, and outcomes in grade III cases were significantly worse than those in grade I and II cases. Preoperative LV diastolic function may be useful in prediction of outcomes after TAVI. (Cardiol J 2019; 26, 1: 29-35)
\end{abstract}

Key words: left ventricular diastolic function, aortic stenosis, transcatheter aortic valve implantation (TAVI), outcomes, prognosis, echocardiography

\section{Introduction}

Some cases of aortic stenosis (AS) are inoperable due to underlying disease, severity and frailty [1-3]. In these cases, the increasingly common use of transcatheter aortic valve implantation (TAVI) may improve prognosis [1, 4]. An increase in AS-induced afterload causes reduced left ventricular (LV) compliance, and elevated LV end-diastolic pressure causes reduced diastolic function, which are causes of a poor prognosis. TAVI reduces the transaortic gradient and relieves afterload, with a resultant improvement in systolic and diastolic function [1, 4-13]. However, the effects of LV diastolic dysfunction on prognosis after TAVI have not been examined. Therefore, the objective of this study was to analyze prognosis based on preoperative LV diastolic function in patients treated with TAVI.

Address for correspondence: Satoru Kayama, MD, Department of Anesthesia, Teikyo University School of Medicine, 2-11-1 Kaga, Itabashi, Tokyo 173-8605, Japan, tel: +81-3-3964-1211, e-mail: masui1213@yahoo.co.jp 


\section{Methods}

\section{Patient population}

A retrospective observational study was conducted in 80 patients who were diagnosed with severe AS and underwent TAVI at Teikyo University Hospital from February 2014 to August 2015. The exclusion criteria included shock, emergency surgery, chronic atrial fibrillation (AF), severe mitral annular calcification, moderate to severe mitral stenosis, and severe mitral regurgitation. Patient background, clinical findings and blood test results were retrieved from electronic medical records (EMRs) and anesthesia charts. Data were collected for age, gender, European System for Cardiac Operative Risk Evaluation II (Euro II) score [5], Logistic Euro (LogEuro) score, Society of Thoracic Surgery (STS) score [6], albumin level [7], renal function based on estimated glomerular filtration rate (eGFR), hypertension, diabetes, dyslipidemia, coronary artery disease (CAD) and percutaneous coronary intervention, chronic obstructive pulmonary disease, and New York Heart Association classification.

\section{Echocardiographic variables}

The following echocardiographic findings were obtained from EMRs: LV ejection fraction (LVEF), E/e', septal e', lateral e', tricuspid regurgitation (TR) velocity, left atrial volume index (LAVI), pulmonary veins: systolic velocity/diastolic velocity ratio $(\mathrm{S} / \mathrm{D}$ ratio) and time difference between duration of pulmonary vein flow and mitral inflow during atrial contraction (Ar-A duration). Echocardiography was performed following American Society of Echocardiography (ASE) guidelines [14, 15]. Preoperative LV diastolic dysfunction was classified into three grades (I, II, and III) based on 2016 ASE Recommendations for Evaluation of Left Ventricular Diastolic Function by Echocardiography [16]. For subjects at the borderline of grades I and II who were difficult to classify with the algorithm, left atrial pressure was assessed with LAVI, S/D ratio and Ar-A duration for classification into grades. Echocardiography in all subjects was performed by two cardiologists at our hospital. In all cases, echocardiography was performed once several days before TAVI subsequent to admission to hospital.

\section{Endpoints}

The endpoints of the study were cardiovascular (CV) events during the follow-up period after discharge, and mortality. The incidences of events on days 180, 360 and 540 were examined. The study was approved by the research ethics committee of the documented hospital. The study protocol conforms to the ethical guidelines of the 1975 Declaration of Helsinki. Exacerbation of heart failure, shift to AF, pneumonia and events including conduction block.

\section{Statistical analysis}

Statistical analyses were performed with EZR (Saitama Medical Center, Jichi Medical University, Saitama, Japan), which is a graphical user interface for R (The R Foundation for Statistical Computing, Vienna, Austria). Continuous variables are expressed as mean \pm standard deviation and were compared by unpaired t test or one-way analysis of variance (ANOVA). Categorical variables are expressed as frequencies (percentages) and compared by the Fisher exact test. Time-to-event analysis was performed using the Kaplan-Meier method and compared between groups by log-rank test. Cox proportional hazards models with best subset regression were used to identify clinical and echocardiographic measurements that were significantly related to cardiac events or death during follow-up. A p value $<0.05$ was considered significant in all analyses.

\section{Results}

\section{Patient characteristics}

The preoperative patient background is shown in Table 1 . The mean age was $85.0 \pm 5.2$ years old and $77.5 \%$ of the subjects were female. Euro II, LogEuro and STS scores were $5.9 \pm 4.6,18.4 \pm$ \pm 9.7 and $8.1 \pm 3.8$, respectively, and LVEF, E/e' and LAVI were $57.5 \pm 10.8 \%, 21.8 \pm 8.6$ and $63.5 \pm$ $\pm 25.1 \mathrm{~mL} / \mathrm{m}^{2}$, respectively. There were no significant differences in age, gender, Euro II, LogEuro, STS, albumin or underlying disease, except for eGFR, CV events, E/e' and LAVI, among patients with LV diastolic dysfunction of grades I, II, and III (Table 2).

\section{Grade classification}

In preoperative echocardiography, LVEF, E/e', LAVI, S/D ratio and Ar-A duration, but not TR velocities, were measured in all subjects. The grade classification was performed based on the algorithm of the 2016 ASE Recommendations for the Evaluation of Left Ventricular Diastolic Function by Echocardiography three subjects however, were difficult to classify and were placed into grade I or II (grade I: 2, grade II: 1) by assessing left 
Table 1. Baseline characteristics of 80 patients in this study.

\begin{tabular}{|c|c|c|c|c|}
\hline Variables & $\begin{array}{l}\text { Overall } \\
(\mathrm{n}=80)\end{array}$ & $\begin{array}{l}\text { Event-free survival } \\
\qquad(n=63)\end{array}$ & $\begin{array}{l}\text { Cardiovascular events } \\
\qquad(\mathrm{n}=17)\end{array}$ & $\mathbf{P}$ \\
\hline Age [years] & $85.0 \pm 5.2$ & $85.3 \pm 4.8$ & $83.9 \pm 6.3$ & 0.3 \\
\hline Female & $62(77.5)$ & $48(76.2 \%)$ & $14(82.4 \%)$ & 0.75 \\
\hline Euro II & $5.9 \pm 4.6$ & $5.4 \pm 4.2$ & $7.9 \pm 5.8$ & 0.043 \\
\hline LogEuro & $18.4 \pm 9.7$ & $17.8 \pm 9.5$ & $20.7 \pm 10.3$ & 0.28 \\
\hline STS & $8.1 \pm 3.8$ & $7.9 \pm 3.7$ & $8.8 \pm 4.2$ & 0.36 \\
\hline Albumin & $3.7 \pm 0.5$ & $3.7 \pm 0.4$ & $3.5 \pm 0.6$ & 0.026 \\
\hline eGFR & $50.2 \pm 17.0$ & $51.0 \pm 17.1$ & $47.5 \pm 16.6$ & 0.46 \\
\hline Hypertension & $60(75 \%)$ & $45(71.4 \%)$ & $15(88.2 \%)$ & 0.21 \\
\hline Diabetes mellitus & $18(22.5 \%)$ & $15(23.8 \%)$ & $3(17.6 \%)$ & 0.75 \\
\hline Dyslipidemia & $47(58.8 \%)$ & $37(58.7 \%)$ & $10(58.8 \%)$ & 1 \\
\hline Coronary artery disease & $12(15.0 \%)$ & $6(9.5 \%)$ & $6(35.2 \%)$ & 0.017 \\
\hline Previous $\mathrm{PCl}$ & $26(32.5 \%)$ & $18(31.0 \%)$ & $8(36.4 \%)$ & 0.16 \\
\hline COPD & $13(16.3 \%)$ & $10(15.8 \%)$ & $3(17.6 \%)$ & 1 \\
\hline \multicolumn{5}{|l|}{ Class NYHA: } \\
\hline 1 & None & None & None & \\
\hline ॥ & $33(41.3 \%)$ & 29 & 4 & \\
\hline III & $35(43.8 \%)$ & 27 & 8 & \\
\hline IV & $12(15.0 \%)$ & 7 & 5 & \\
\hline Class NYHA III-IV & $47(58.8 \%)$ & $34(54.0 \%)$ & $13(76.5 \%)$ & 0.11 \\
\hline \multicolumn{5}{|l|}{ Diastolic dysfunction: } \\
\hline Grade I & $19(23.8 \%)$ & 17 & 2 & \\
\hline Grade II & $49(61.3 \%)$ & 41 & 8 & \\
\hline Grade III & $12(15.0 \%)$ & 5 & 7 & \\
\hline LVEF & $57.5 \pm 10.8$ & $57.9 \pm 10.2$ & $56.1 \pm 13.1$ & 0.55 \\
\hline$E / e^{\prime}$ & $21.8 \pm 8.6$ & $22.3 \pm 8.9$ & $19.6 \pm 7.1$ & 0.29 \\
\hline LAVI & $63.5 \pm 25.1$ & $61.0 \pm 23.5$ & $72.6 \pm 28.5$ & 0.093 \\
\hline
\end{tabular}

Continuous variables are shown as mean \pm standard deviation, and categorical variables as frequencies (\%); Euro II - European System for Cardiac Operative Risk Evaluation Score II; LogEuro - Logistic Euro score; STS - Society of Thoracic Surgeons score; eGFR - estimated glomerular filtration rate; $\mathrm{PCl}$ - percutaneous coronary intervention; COPD — chronic obstructive pulmonary disease; NYHA - New York Heart Association; LVEF — left ventricular ejection fraction; LAVI — left atrial volume index

atrial pressure in accordance with LAVI, S/D and Ar-A duration.

\section{Complications during TAVI}

Complications developing during TAVI included hemorrhage from a puncture site in 2 patients; femoral artery occlusion, oozing from the left ventricle after TAVI, mitral valve prolapse, cardiac tamponade after TAVI, right ventricular injury caused by a pacing wire, and stenosis due to calcified plaque in the entrance of the left coronary artery after TAVI in 1 patient and complete atrioventricular block after TAVI in 4 patients. No patients died during surgery and the follow-up survey after discharge from hospital was conducted in all subjects.

\section{Cardiovascular events}

The average follow-up was 529 days (interquartile range [IQR] 358-741 days). Cardiovascular events during follow-up occurred in 17 of 80 subjects, and were exacerbated by heart failure in all 17 subjects. Of these subjects, 4 developed heart failure with AF. Onset of heart failure with AF was defined as CV events when NT-pro-B-type natriuretic peptide was higher during the observation of period after discharge than that at the time of discharge and the patients had clinical symptoms including dyspnea and pedal edema, consequently, they were diagnosed with $\mathrm{AF}$-induced heart failure. Heart failure was the cause of death in all 6 subjects who died (grade I: 1 , grade II: 5 , grade III: 0 ). 
Table 2. Baseline grades for severity of diastolic dysfunction.

\begin{tabular}{|c|c|c|c|c|}
\hline Variables & Grade I (n = 19) & Grade II (n = 49) & Grade III (n = 12) & $\mathbf{P}$ \\
\hline Age [years] & $85.9 \pm 4.7$ & $85.4 \pm 4.7$ & $82.2 \pm 7.0$ & 0.11 \\
\hline Female & $13(68.4 \%)$ & $37(75.5 \%)$ & $12(100 \%)$ & 0.1 \\
\hline Euro II & $5.8 \pm 5.2$ & $5.6 \pm 4.1$ & $7.3 \pm 5.8$ & 0.5 \\
\hline LogEuro & $16.3 \pm 8.4$ & $19.3 \pm 10.7$ & $18.2 \pm 6.9$ & 0.53 \\
\hline STS & $7.4 \pm 3.1$ & $8.3 \pm 3.7$ & $8.4 \pm 5.2$ & 0.69 \\
\hline Albumin & $3.8 \pm 0.3$ & $3.7 \pm 0.5$ & $3.7 \pm 0.6$ & 0.73 \\
\hline eGFR & $56.8 \pm 19.0$ & $49.8 \pm 16.3$ & $41.4 \pm 12.2$ & 0.044 \\
\hline Hypertension & $14(73.7 \%)$ & $37(75.5 \%)$ & $9(75.0 \%)$ & 1 \\
\hline Diabetes mellitus & $5(26.3 \%)$ & $12(24.5 \%)$ & $1(8.3 \%)$ & 0.51 \\
\hline Dyslipidemia & $11(57.9 \%)$ & $27(55.1 \%)$ & $9(75.0 \%)$ & 0.5 \\
\hline Coronary artery disease & $3(15.8 \%)$ & $7(14.3 \%)$ & $2(16.7 \%)$ & 1 \\
\hline Previous PCI & $7(36.8 \%)$ & $17(34.7 \%)$ & $2(16.7 \%)$ & 0.49 \\
\hline COPD & $4(21.1 \%)$ & $9(18.4 \%)$ & $0(0 \%)$ & 0.29 \\
\hline Class NYHA III-IV & $10(52.6 \%)$ & $26(53.1 \%)$ & $9(75.0 \%)$ & 0.4 \\
\hline Cardiovascular events & $2(10.5 \%)$ & $8(16.3 \%)$ & $7(58.3 \%)$ & $<0.001$ \\
\hline LVEF & $58.9 \pm 7.8$ & $57.3 \pm 11.8$ & $56.3 \pm 11.3$ & 0.78 \\
\hline$E / e^{\prime}$ & $14.2 \pm 4.8$ & $23.9 \pm 8.4$ & $25.1 \pm 6.9$ & $<0.001$ \\
\hline LAVI & $43.7 \pm 13.8$ & $65.3 \pm 21.1$ & $90.0 \pm 29.5$ & $<0.001$ \\
\hline
\end{tabular}

Continuous variables are shown as mean \pm standard deviation, and categorical variables as frequencies (\%); Euro II - European System for Cardiac Operative Risk Evaluation Score II; LogEuro - Logistic Euro score; STS - Society of Thoracic Surgeons score; eGFR - estimated glomerular filtration; PCl — percutaneous coronary intervention; COPD - chronic obstructive pulmonary disease; NYHA - New York Heart Association; LVEF — left ventricular ejection fraction; LAVI — left atrial volume index

Non-CV events after discharge included pneumonia and 3-degree block (after 314 days) in 1 subject each and there were no deaths.

Some patients did not undergo echocardiography because they could not visit the hospital due to being elderly or having to travel a long distance, and some were excluded from follow-up due to CV events during follow-up after TAVI. In the survey of prognoses, no patient was lost to follow-up because prognoses were determined by interview when visiting the hospital or telephone calls to patients or their families.

Albumin $(3.5 \pm 0.6$ vs. $3.7 \pm 0.4 \mathrm{~g} / \mathrm{dL}, \mathrm{p}=$ $=0.026)$, Euro II score (7.8 \pm 5.8 vs. $5.4 \pm 4.2$, $\mathrm{p}=0.043)$ and $\mathrm{CAD}(35.2 \%$ vs. $9.5 \%, \mathrm{p}=0.017)$ were significantly related to CV events (Table 1 ). In grades I, II and III, the 180-day event-free survival (EFS) rates were $89.5 \%, 89.5 \%$ and $46.9 \%$, the 360 -day EFS rates were $89.5 \%, 89.5 \%$ and $37.5 \%$, and the 540-day EFS rates were $89.5 \%, 82.3 \%$ and $37.5 \%$, respectively. In grade III cases, the median EFS was 180 days (Fig. 1) and the prognosis based on EFS rates which were significantly worse than for grades I and II $(p=0.00013)$. Events occurred more frequently in cases with lower albumin $(<3.6$ vs. $\geq 3.6 \mathrm{~g} / \mathrm{dL}, \mathrm{p}=0.0012)$, and $\mathrm{CAD}(+)$ vs. $\mathrm{CAD}(-), \mathrm{p}=0.0049$; Fig. 2).

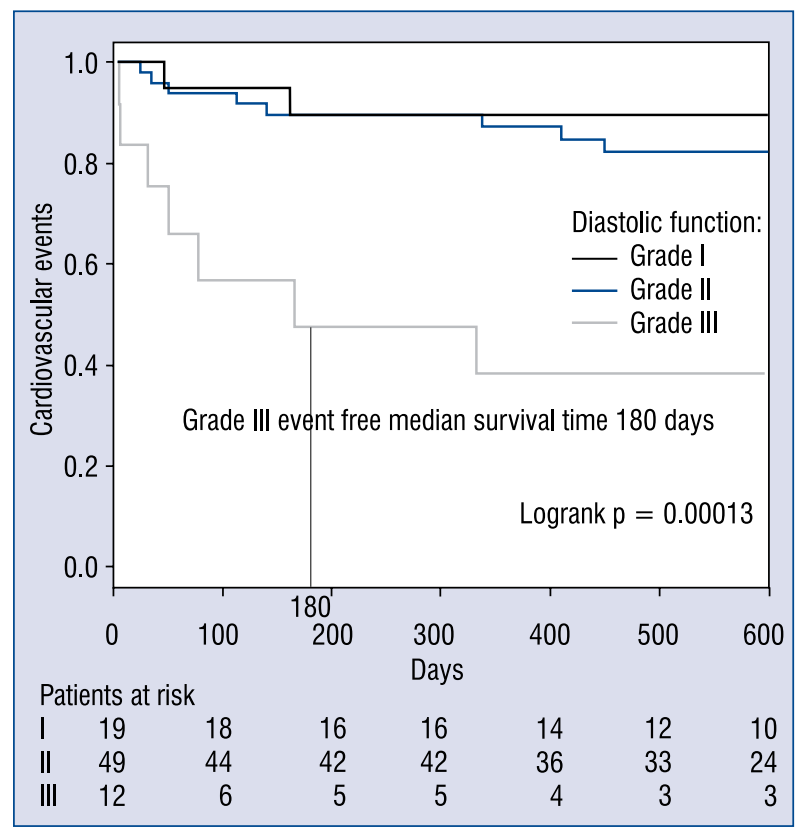

Figure 1. Kaplan-Meier event-free survival (EFS) rates for patients with diastolic function of grade I ( $n=19)$, grade II ( $n=49)$, and grade III $(n=12)$. The grade III median EFS time was 180 days. In grades I, II and III, the EFS rates were $89.5 \%, 89.5 \%$, and $46.9 \%$ at 180 days; $89.5 \%, 89.5 \%$, and $37.5 \%$ at 360 days; and $89.5 \%$, $82.3 \%$, and $37.5 \%$ at 540 days, respectively. 


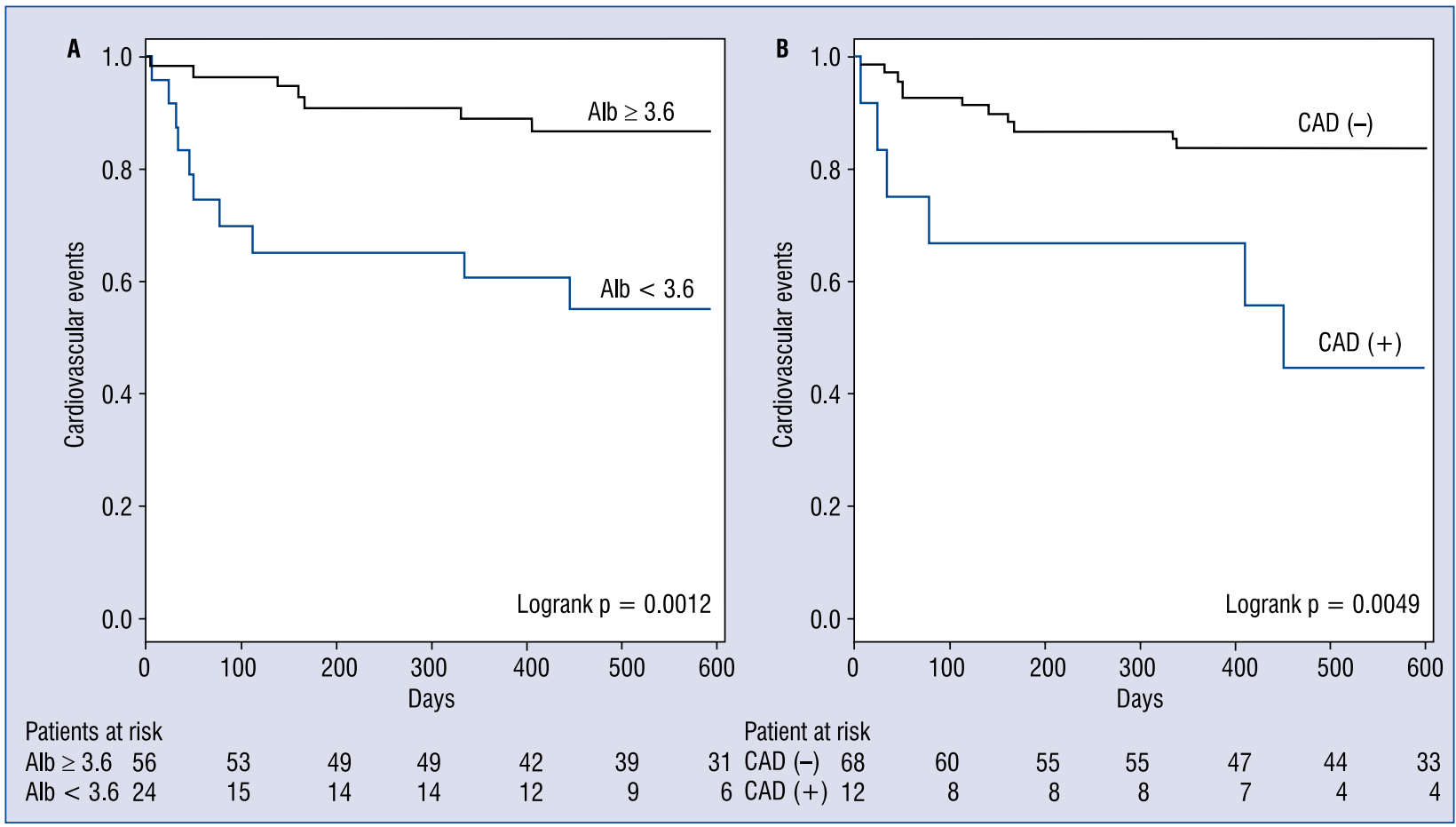

Figure 2. Kaplan-Meier event free survival rates for cases with $(A)$ albumin $(A l b)<3.6 \mathrm{~g} / \mathrm{dL}$ vs. $\geq 3.6 \mathrm{~g} / \mathrm{dL},(B) \mathrm{CAD}(+)$ vs. CAD(-); CAD - coronary artery disease.

Table 3. Multivariate analysis of clinical and echocardiographic predictors of events.

\begin{tabular}{lcccccc}
\hline \multirow{2}{*}{ Variables } & \multicolumn{2}{c}{ Univariate analysis } & & \multicolumn{3}{c}{ Multivariate analysis } \\
\cline { 2 - 3 } & HR $(95 \% \mathrm{CI})$ & $\mathbf{P}$ & & HR $(95 \% \mathrm{CI})$ & $\mathrm{P}$ \\
\hline Diastolic dysfunction & $3.84(1.66-8.84)$ & 0.0016 & & $3.99(1.35-11.8)$ & 0.012 \\
Low albumin $(<3.6 \mathrm{~g} / \mathrm{dL})$ & $4.36(1.65-11.49)$ & 0.0029 & & $4.73(1.42-15.8)$ & 0.012 \\
Coronary artery disease & $3.78(1.40-10.24)$ & 0.0089 & & & & \\
\hline
\end{tabular}

$\mathrm{Cl}$ - confidence interval; $\mathrm{HR}$ - hazard ratio

\section{Predictors of medium and long-term prognosis}

In univariate analysis, $\mathrm{LV}$ diastolic dysfunction (hazard ratio [HR] 3.84, $\mathrm{p}=0.0016$ ), low albumin $(<3.6 \mathrm{~g} / \mathrm{dL})(\mathrm{HR} 4.36, \mathrm{p}=0.0029)$, and CAD (HR 3.78, $\mathrm{p}=0.0089$ ) were associated with CV events or mortality. In multivariate Cox regression analysis, LV diastolic dysfunction (HR 3.99, $\mathrm{p}=$ $=0.012)$ and low albumin (HR 4.73, $\mathrm{p}=0.012$ ) were independent predictors of a poor outcome during the follow-up period (Table 3 ).

\section{Discussion}

Technical improvements and advances in devices due to accumulated data have contributed to better outcomes of TAVI; however, problems remain regarding preoperative evaluation and postoperative complications. In this study, the relationship between preoperative LV diastolic function and postoperative complications in patients treated with TAVI was investigated. Pre-diastolic function grade was found to be strongly associated that a higher grade of preoperative LV diastolic dysfunction and with poorer EFS. Especially, events in grade III cases which occurred earlier and at a higher incidence than those for grades I and II. A Cox proportional hazards model indicated that preoperative LV function is an independent risk factor for postoperative CV events. Preoperative LV diastolic dysfunction has been shown to affect EFS after CV surgery [17]. TAVI is a brief and 
less invasive procedure that improves systolic and diastolic function at an earlier stage compared with surgical aortic valve replacement $[4,18]$. The function of the LV system, including the left atrium, and right ventricular function recover early after TAVI and diastolic function is improved $[4,8]$, while AS-induced cardiac hypertrophy is improved over the medium to long-term, this further improves LV function $[4,13,18,19]$.

Albumin has been proposed to be a prognostic factor for postoperative mortality and complications [7, 20]. Yamamoto et al. [7] indicated that hypoalbuminemia was a predictor for mortality and complications after TAVI, and 30-day mortality was significantly higher in patients with albumin $<3.5 \mathrm{~g} / \mathrm{dL}$. The results of this study also showed that EFS after TAVI was significantly lower in subjects with albumin $<3.6 \mathrm{~g} / \mathrm{dL}$, which closely matches the results of Yamamoto et al. [7].

Of note is an algorithm proposed by Nagueh et al. [16] which was used for detection and severity classification of LV diastolic dysfunction into grades. This algorithm is simple to use for detection, but when the grade was difficult to determine because of the border zone (grade I or II), it was based on other echocardiographic findings, such as LAVI, S/D ratio and Ar-A duration. All subjects classified using other echocardiographic findings had no data for TR, due to a failure of TR velocity measurement. LAVI and S/D are related to mean left atrial pressure, and Ar-A duration is related to LV end-diastolic pressure [16]; therefore, increased left atrial pressure was assessed using these data and subjects were classified into grades I or II.

The most important finding in this study was that EFS rate in patients with preoperative LV diastolic dysfunction of grade III who were significantly worse than those in patients with grades I and II. The median event occurrence period was 180 days in grade III, which suggests that patients in grade III specially take care of follow-up after TAVI. Patients who complicate with CAD, which was found to be a risk factor in this study, may have further adverse outcomes. Use of TAVI has been slowly increasing, therefore some indices including LV diastolic function may be important for preoperative evaluation before TAVI.

\section{Limitations of the study}

The major limitations of the study were the retrospective observational design and the inclusion of some subjects in whom detailed courses could not be followed for an extended period. The guidelines for LV diastolic function did not allow for some subjects to be classified into a grade using the algorithm alone. These subjects were classified by estimating increased left atrial pressure using other indicators; however, the accuracy of this method is unclear and the assigned grade is uncertain. Detailed courses were not found in all EMRs because elderly patients could not undergo echocardiography or a full interview during the observational period. In echocardiography, detailed data were available before TAVI, whereas postoperative data collected during follow-up had many missing values because some patients could not undergo echocardiography due to their condition. Consequently, changes in the grade of LV diastolic function from prior to and after TAVI or postoperative echocardiographic findings in follow up could not be verified. There was no postoperative mortality in 6 subjects in grade III, showing no tendency for worsening of outcomes at a higher grade. However, the number of subjects was insufficient to evaluate the significance of mortality data. Euro II, LogEuro and STS scores were noted and used as indicators for preoperative risk assessment in CV surgery. In this study, significant differences in Euro II scores alone were found among cases with different LV diastolic dysfunction. Some studies indicate overestimation of these risk scores [21, 22], but it is unclear why only Euro II scores were significant in this study. However, it was noted that Euro II, LogEuro and STS scores are predictors of mortality risk after conventional CV surgeries, these scores may not be applicable to clinical status and performance of TAVI in extremely elderly patients.

\section{Conclusions}

Postoperative events after TAVI occurred more frequently in patients with advanced preoperative LV diastolic dysfunction, as found after other CV surgery. Events in cases with grade III LV diastolic dysfunction occurred earlier and at a significantly higher incidence than those in grades I and II. Therefore, diastolic function may be a useful indicator for prediction of outcomes after TAVI. A large-scale prospective study is required to validate these findings. Present results show that postoperative outcomes after TAVI are influenced by several factors and these findings make the study particularly valuable in identifying patients who will benefit most from TAVI.

Conflict of interest: None declared 


\section{References}

1. Costantino MF, Galderisi M, Dores E, et al. Parallel improvement of left ventricular geometry and filling pressure after transcatheter aortic valve implantation in high risk aortic stenosis: comparison with major prosthetic surgery by standard echo Doppler evaluation. Cardiovasc Ultrasound. 2013; 11: 18, doi: 10.1186/1476-7120-11-18, indexed in Pubmed: 23731705.

2. Van Mieghem NM, Chieffo A, Dumonteil N, et al. Trends in outcome after transfemoral transcatheter aortic valve implantation. Am Heart J. 2013; 165(2): 183-192, doi: 10.1016/j. ahj.2012.11.002, indexed in Pubmed: 23351821.

3. Lichtenstein SV, Cheung A, Ye J, et al. Transapical transcatheter aortic valve implantation in humans: initial clinical experience. Circulation. 2006; 114(6): 591-596, doi: 10.1161/CIRCULATIONAHA.106.632927, indexed in Pubmed: 16880325.

4. Sarı C, Aslan AN, Baştuğ S, et al. Immediate recovery of the left atrial and left ventricular diastolic function after transcatheter aortic valve implantation: A transesophageal echocardiography study. Cardiol J. 2016; 23(4): 449-455, doi: 10.5603/ CJ.a2016.0030, indexed in Pubmed: 27296155.

5. Rosato S, Santini F, Barbanti M, et al. OBSERVANT Research Group. Transcatheter Aortic Valve Implantation Compared With Surgical Aortic Valve Replacement in Low-Risk Patients. Circ Cardiovasc Interv. 2016; 9(5): e003326, doi: 10.1161/CIRCINTERVENTIONS.115.003326, indexed in Pubmed: 27154298.

6. Leon MB, Smith CR, Mack M, et al. Transcatheter aortic-valve implantation for aortic stenosis in patients who cannot undergo surgery. N Engl J Med. 2010; 363(17): 1597-1607, doi: 10.1056/ NEJMoa1008232, indexed in Pubmed: 20961243.

7. Yamamoto M, Shimura T, Kano S, et al. Prognostic Value of Hypoalbuminemia After Transcatheter Aortic Valve Implantation (from the Japanese Multicenter OCEAN-TAVI Registry). Am J Cardiol. 2017; 119(5): 770-777, doi: 10.1016/j.amjcard.2016.11.019, indexed in Pubmed: 28017301.

8. Zhao Y, Lindqvist P, Nilsson J, et al. Trans-catheter aortic valve implantation--early recovery of left and preservation of right ventricular function. Interact Cardiovasc Thorac Surg. 2011; 12(1): 35-39, doi: 10.1510/icvts.2010.251348, indexed in Pubmed: 20956401.

9. Lancellotti P, Magne J, Donal E, et al. Clinical outcome in asymptomatic severe aortic stenosis: insights from the new proposed aortic stenosis grading classification. J Am Coll Cardiol. 2012; 59(3): 235-243, doi: 10.1016/j.jacc.2011.08.072, indexed in Pubmed: 22240128.

10. Holme I, Pedersen TR, Boman K, et al. A risk score for predicting mortality in patients with asymptomatic mild to moderate aortic stenosis. Heart. 2012; 98(5): 377-383, doi: 10.1136/ heartjnl-2011-300475, indexed in Pubmed: 22155702.

11. Gonçalves A, Marcos-Alberca P, Almeria C, et al. Acute left ventricle diastolic function improvement after transcatheter aortic valve implantation. Eur J Echocardiogr. 2011; 12(10): 790-797, doi: 10.1093/ejechocard/jer147, indexed in Pubmed: 21865229.
12. Clavel MA, Webb JG, Rodés-Cabau J, et al. Comparison between transcatheter and surgical prosthetic valve implantation in patients with severe aortic stenosis and reduced left ventricular ejection fraction. Circulation. 2010; 122(19): 1928-1936, doi: 10.1161/ CIRCULATIONAHA.109.929893, indexed in Pubmed: 20975002.

13. Vizzardi E, D'Aloia A, Fiorina C, et al. Early regression of left ventricular mass associated with diastolic improvement after transcatheter aortic valve implantation. J Am Soc Echocardiogr. 2012; 25(10): 1091-1098, doi: 10.1016/j.echo.2012.06.010, indexed in Pubmed: 22819229.

14. Zamorano JL, Badano LP, Bruce C, et al. EAE/ASE recommendations for the use of echocardiography in new transcatheter interventions for valvular heart disease. J Am Soc Echocardiogr. 2011; 24(9): 937-965, doi: 10.1016/j.echo.2011.07.003, indexed in Pubmed: 21867869.

15. Hahn RT, Abraham T, Adams MS, et al. Guidelines for performing a comprehensive transesophageal echocardiographic examination: recommendations from the American Society of Echocardiography and the Society of Cardiovascular Anesthesiologists. J Am Soc Echocardiogr. 2013; 26(9): 921-964, doi: 10.1016/j. echo.2013.07.009, indexed in Pubmed: 23998692.

16. Nagueh SF, Smiseth OA, Appleton CP, et al. Recommendation for the evaluation of left ventricular diastolic function by echocardiography: An update from the American Society of Echocardiography and the European Association of Cardiovascular Imaging. Eur Heart J Cardiovasc Imaging. 2016; 17(12): 1321-1360.

17. Kaw R, Hernandez AV, Pasupuleti V, et al. Effect of diastolic dysfunction on postoperative outcomes after cardiovascular surgery: A systematic review and meta-analysis. J Thorac Cardiovasc Surg. 2016; 152(4): 1142-1153, doi: 10.1016/j.jtcvs.2016.05.057, indexed in Pubmed: 27364601.

18. La Manna A, Sanfilippo A, Capodanno D, et al. Left ventricular reverse remodeling after transcatheter aortic valve implantation: a cardiovascular magnetic resonance study. J Cardiovasc Magn Reson. 2013; 15: 39, doi: 10.1186/1532-429X-15-39, indexed in Pubmed: 23692630.

19. van Straten AHM, Soliman Hamad MA, Peels KCH, et al. Increased septum wall thickness in patients undergoing aortic valve replacement predicts worse late survival. Ann Thorac Surg. 2012; 94(1): 66-71, doi: 10.1016/j.athoracsur.2012.03.027, indexed in Pubmed: 22607789.

20. Gibbs J, Cull W, Henderson W, et al. Preoperative serum albumin level as a predictor of operative mortality and morbidity: results from the National VA Surgical Risk Study. Arch Surg. 1999; 134(1): 36-42, indexed in Pubmed: 9927128.

21. Sündermann S, Dademasch A, Rastan A, et al. Comprehensive assessment of frailty for elderly high-risk patients undergoing cardiac surgery. Eur J Cardiothorac Surg. 2011; 39(1): 33-37, doi: 10.1016/j.ejcts.2010.04.013, indexed in Pubmed: 20627611.

22. Basraon J, Chandrashekhar YS, John R, et al. Comparison of risk scores to estimate perioperative mortality in aortic valve replacement surgery. Ann Thorac Surg. 2011; 92(2): 535-540, doi: 10.1016/j. athoracsur.2011.04.006, indexed in Pubmed: 21704289. 